\title{
Providing Patients with Implantable Cardiac Device Data through a Personal Health Record: A Qualitative Study
}

\author{
Carly N. Daley ${ }^{1,2}$ Elizabeth M. Chen ${ }^{1,3}$ Amelia E. Roebuck ${ }^{1}$ Romisa Rohani Ghahari ${ }^{1}$ Areej F. Sami ${ }^{4}$ \\ Cayla G. Skaggs ${ }^{1}$ Maria D. Carpenter ${ }^{1}$ Michael J. Mirro ${ }^{1,4}$ Tammy R. Toscos ${ }^{1,2}$
}

${ }^{1}$ Department of Research Informatics, Parkview Health System, Mirro

Address for correspondence Carly N. Daley, MS, Parkview Mirro Center for Research and Innovation, Fort Wayne, Indiana, United States

2 Department of BioHealth Informatics, Indiana University School of Center for Research and Innovation, 10622 Parkview Plaza Dr., Fort Informatics and Computing, Indianapolis, Indiana, United States

${ }^{3}$ Marian University College of Osteopathic Medicine, Indianapolis, Wayne, IN 46845, United States (e-mail: carly.daley@parkview.com).

Indiana, United States

${ }^{4}$ Department of Medicine, Indiana University School of Medicine, Indianapolis, Indiana, United States

Appl Clin Inform 2017;8:1106-1116.

\section{Abstract}

Keywords

- personal health records

- remote monitoring

- implantable cardioverter defibrillators

- patient engagement

- qualitative
Background Patients at risk for sudden cardiac death or having suffered cardiac arrest may receive an implantable cardioverter defibrillator (ICD). This device provides monitoring and therapy for life-threatening heart rhythms. Remote monitoring of ICDs has decreased the time between abnormal heart rhythm events and clinic follow-up. Currently, the data transmitted from the device are reviewed and stored by the clinic, but patients do not have access to the data. While connecting patients directly with their ICD data has potential to enhance engagement in their care and improve health outcomes, patient attitudes and perceptions about receiving ICD data have not been explored.

Objective This research is the first demonstration of delivering ICD data to patients through a personal health record (PHR) using a novel technical framework. The objective of this study was to use a PHR interface as a technology probe to explore patients' experiences with directly receiving their ICD data from remote monitoring. Methods We enrolled 21 patients with an ICD undergoing remote monitoring at a large outpatient cardiology clinic in Indiana, United States. Participants received their ICD data from remote monitoring through a PHR over 3 months. In-depth, semistructured interviews were conducted at 3 months and analyzed using thematic analysis. Results Participants were 36 to 86 years old, mean age (SD) of 67 (14) years, predominately male (76\%), and white (95\%). Key themes were ICD questions and concerns, experiences with remote monitoring, PHR use, and feedback about the ICD data summary. The findings showed that overall, patients desired information that provides reassurance, is easy to understand, and is presented in a meaningful way.

Conclusion Sharing ICD data from remote monitoring requires adequate context and scaffolding to support patient understanding. Engaging patients with information that is useful and valuable to them through a PHR may require appropriate and individualized tailoring of information. received

June 6, 2017

accepted after revision

October 5, 2017
Copyright @ 2017 Schattauer
DOI https://doi.org/ 10.4338/ACI-2017-06-RA0090 .

ISSN 1869-0327. 


\section{Background and Significance}

The development of remote monitoring technology within the past two decades has improved the process of checking heart and device status for patients with implantable cardioverter defibrillators (ICDs). ${ }^{1}$ Hundreds of data elements related to heart function and device status are transmitted from the ICD, reviewed by clinicians, and stored in the patient medical record. Patients receive follow-up phone calls or letters from the clinic indicating that they need to come in to the clinic or their status is essentially normal; however, patients do not have direct access to the data. Patients have experienced frustration and anxiety over their rights to their own ICD data, the lack of feedback for reassurance, and uncertainty about what type of information is transmitted. ${ }^{2-5}$ In a study examining ICD patients' satisfaction with remote monitoring follow-up, $95 \%$ of patients undergoing remote monitoring $(N=385)$ were satisfied with their current follow-up protocol. ${ }^{6}$ Still, $84 \%$ wanted more detail in the response, and $21 \%$ desired a faster response after the transmission.

Remote monitoring requires having a transmitter box in the patient's home and using a landline or cellular technology to transmit the data. Research shows that the remote monitoring system can be intimidating to patients, make them feel as though they are being watched, and contribute to fear of losing face-to-face interactions with physicians. ${ }^{7,8}$ Prior research has shown that sharing clinical data with patients through a personal health record (PHR) may increase patients' awareness and engagement. ${ }^{9}$ This suggests that there may be potential to increase understanding of the device and remote monitoring by sharing ICD data with patients. Sharing data and information with patients may increase transparency and understanding while also mitigating some of the anxieties generated from the unknown or misunderstood aspects of remote monitoring. We believe that delivering ICD data to patients could enhance patient engagement, or the "process of active participation where users invest time and attention." ${ }^{10}$ To explore patients' perceptions and attitudes toward receiving complex remote monitoring data, we developed a mechanism to deliver ICD data to a PHR interface for patients to view. Using a qualitative, exploratory approach, we gathered insights from patients to understand their attitudes and perceptions about receiving ICD data from remote monitoring in a PHR.

\section{Significance of the Project}

This project is the first demonstration of delivering ICD data from the device to a PHR in a patient-facing interface. ${ }^{11} \mathrm{We}$ believe that providing patients with their ICD data from remote monitoring has the potential to improve engagement and satisfaction, which may enhance adherence to remote monitoring, as suggested in prior research. ${ }^{6-9}$ We recognize that experiential problems exist with remote monitoring; ${ }^{7,8}$ however, we believe that increasing patient understanding can generate trust in the system and further empower patients and alleviate concerns. Providing patients with data from remote monitoring in a meaningful way also has the potential to enhance communication during clinic visits.
In this article, we describe themes uncovered through indepth interviews with 21 patients after they completed our 3-month technology trial. By looking deeply into patients' experiences with the ICD data summary, including perceptions related to their ICD and remote monitoring, we uncovered insights into patients' attitudes and perceptions about receiving ICD data in a PHR.

\section{Objective}

The objectives of our study were to explore:

1. Patients' attitudes and perceptions about receiving ICD data through a PHR.

2. Insights about how to present the data in a meaningful way.

\section{Methods}

This qualitative, exploratory study involved a 3-month technology trial where participants received their ICD remote monitoring transmission data in a PHR. A PHR is a web-based tool for storing and sharing health information. By definition, a PHR is more than a portal; patients can receive and exchange information such as laboratory results and also use it to enter data that they generated themselves. ${ }^{12}$ Prior to participant enrollment, a novel widget display for ICD data was designed and developed within the PHR interface specifically for this study. To test the data flow and provide participants with their own ICD data in a PHR, we used an innovative and secure technical framework for delivering cardiac device data.

\section{Personal Health Record Intervention}

The ICD data summary appeared as a widget embedded in the member summary page of the PHR and included a hyperlink to a separate page with more details from the report. The ICD data summary included battery status (in months remaining), lead status (in ohms), and ventricular therapies including number of shocks or antitachycardia pacing episodes (-Fig. 1). These three observations were identified as high value data elements from the Heart Rhythm Society (verbal conversation with Dr. Michael Mirro and Dr. David Slotwiner, 2013), based on the understanding that patients with cardiac devices generally want to know (1) the battery life of their device, (2) if their lead wires are functioning properly, and (3) if their device performed any pacing therapy or delivered shocks for ventricular tachycardia. The data have the potential to reassure patients that their device and remote monitor are working and that their heart rhythm is normal, as well as help with timely decision making (e.g., to schedule follow-up appointment for device adjustment).

The innovative method of integrating the device data involved a technical framework called the Implantable Cardiac Device Observation (IDCO) profile that supports interoperability across electronic platforms. ${ }^{13}$ A multidisciplinary team including engineers from the device vendor company, PHR developers, hospital interface specialists, and research and cardiology clinic staff collaborated to construct the PHR interface and establish connectivity from the remote monitoring database to the PHR. 


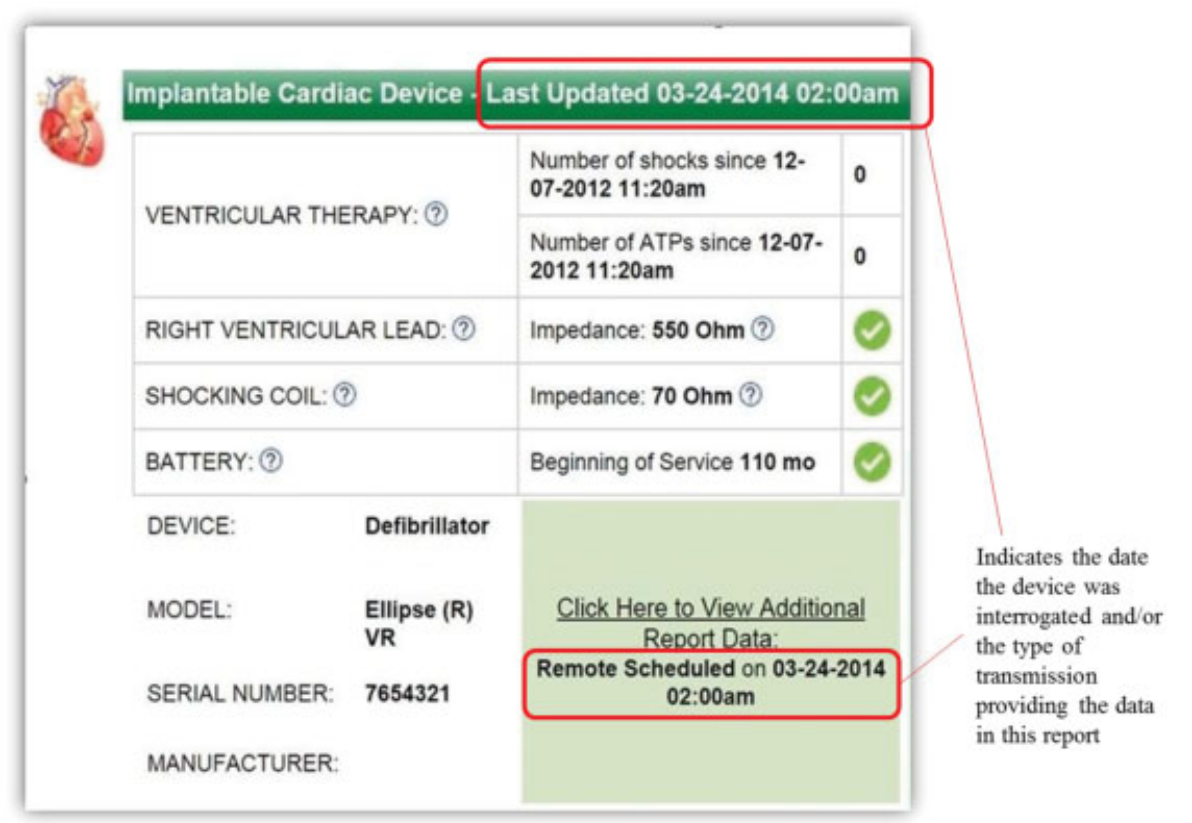

Fig. 1 The patient notification summary of implantable cardioverter defibrillator data appeared on the member summary page of the personal health record. By clicking on the hyperlink, "Click here to View Additional Report Data," participants could access a report with more data from the transmission including lead information, device settings and measurements, capacitor charge information, rates, and other statistics.

There was no patient involvement in the design of the interface; rather it was designed by technologists, clinicians, and researchers and used as a probe to elicit responses from participants about receiving ICD data.

\section{Setting}

The study was conducted at a large cardiology clinic in Indiana, United States. The clinic is part of a health system that serves a population of approximately 890,000, with eight hospitals located in six counties in northeast Indiana and Ohio. The cardiology clinic has over 51,000 patient encounters annually among 15 offices located throughout the service area. Study visits took place in the cardiology ambulatory clinic office.

\section{Participants, Recruitment, and Enrollment}

A convenience sample of 21 patients was enrolled in this study from January through March 2014. The inclusion criteria for the study required that patients had an ICD device from one vendor and were actively enrolled in remote monitoring. Approximately 600 patients met these criteria, and only those with single or dual chamber ICDs were contacted for recruitment (see - Fig. 2 for detailed description).

After completing the informed consent process, participants registered for a PHR account and were shown how to access their PHR on a desktop computer in a private room of the cardiology outpatient clinic. This particular PHR had been offered to patients at this clinic beginning in 2005; however, none of the participants had active accounts and all registered for an account as part of the enrollment process. During the 3-month study period, transmission reports were exported from the database to the participants' chart in the electronic health record (EHR) and then to their personal health record (PHR) in near real time as discrete data (-Fig. 3). Transmis- sions occurred as either scheduled, alert-initiated, or patientinitiated transmissions. Participants had anywhere from one to three transmissions during the study period. Additional details regarding the development of the ICD data summary and data flow can be found in an earlier publication. ${ }^{11}$

\section{Participant Interviews}

A qualitative study design was chosen to understand the complex factors that affect patients' perceptions about receiving their ICD data from remote monitoring. Semistructured, in-depth interviews covered a range of aspects related to the ICD data summary, from the participants' experience with remote monitoring as well as their experience receiving the ICD data summary during the trial. Consistent with established qualitative research methods, ${ }^{14}$ the in-depth, semistructured interviews used broad, open-ended questions to elicit personal thoughts and experiences regarding remote monitoring and the ICD data summary. See - Appendix A for the interview guide. Each interview was digitally recorded and lasted approximately 30 to 40 minutes.

The interview included a common usability exercise, the Think Aloud Technique. ${ }^{15}$ The participants interacted with a mock PHR account, performing specified tasks while expressing their thoughts throughout the interaction. The process helped identify how the participants interacted with the PHR. Study participants were encouraged to reflect and share their personal experiences using their PHR and receiving remote monitoring data during the study.

\section{Interview Analysis}

The interviews were conducted by one researcher and assistant who took observation notes. The interviews were recorded and transcribed. The interviewer had a background in psychology 


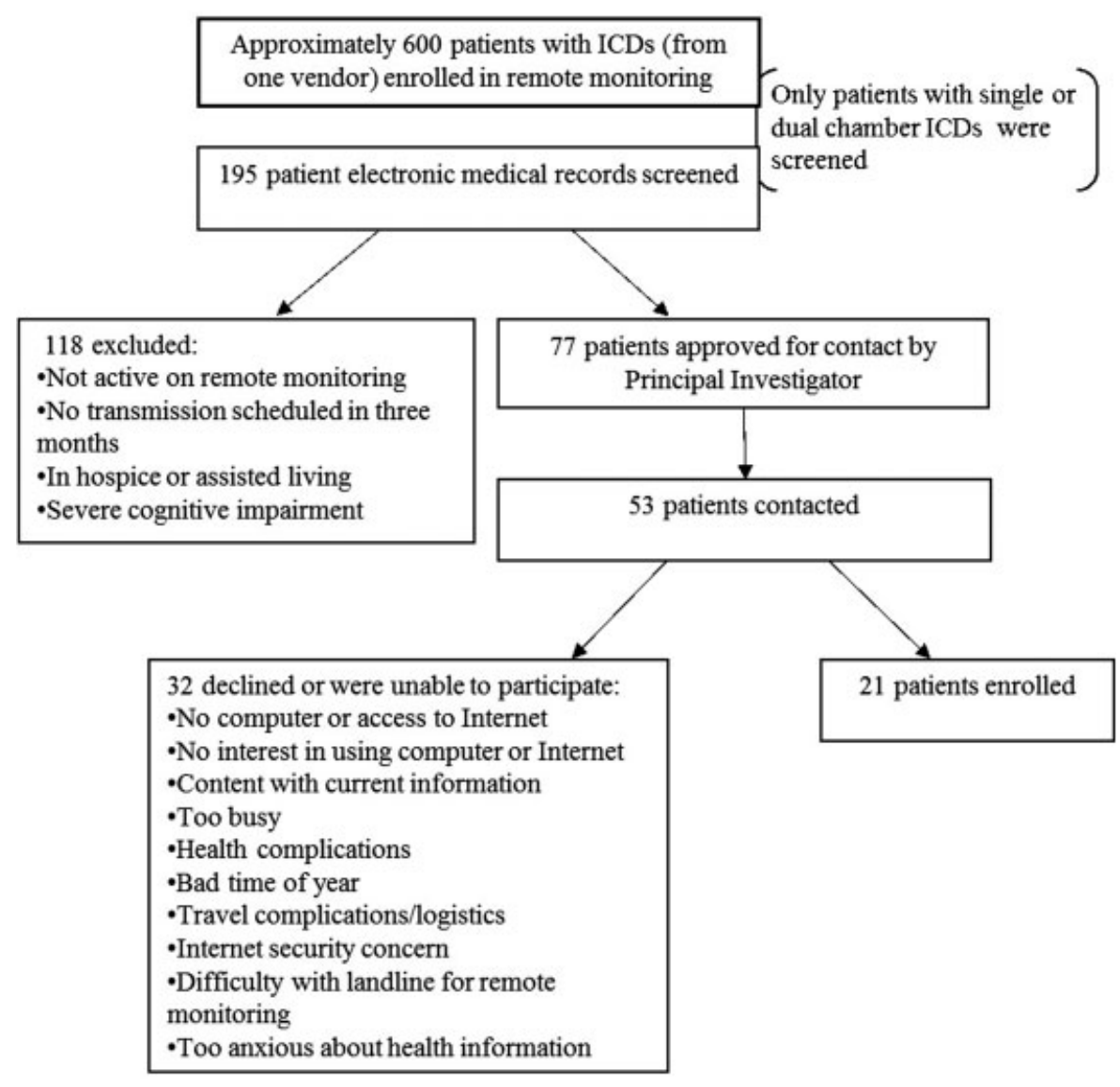

Fig. 2 Flow diagram illustrating the screening, recruitment, and enrollment numbers. Reasons given by patients for declining participation in the study included computer and Internet access, logistical issues, and personal health concerns. ICD, implantable cardioverter defibrillator.

and health informatics and coordinated the study, including the development of the PHR interface. The primary interview analysis team, led by the interviewer, included three informatics research assistants, an informatics research nurse coordinator, and one research scientist. The team members were not involved in the design of the study, which helped balance biased interpretations of the data by the interviewer who was deeply involved in the study development. A preliminary set of codes was developed as the team read and discussed transcripts. Each team member then coded a transcript individually based on the preliminary set of codes, followed by group comparison of coded segments and discussion. ${ }^{16}$ The researchers compared text with previous segments that had been assigned the same code to refine and/or establish new codes. ${ }^{17}$ Final agreement on the codes was established once no new codes were generated, and the group coded transcripts with

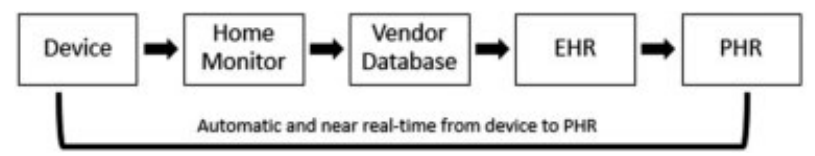

Fig. 3 Data flow from implantable cardiac device to the PHR. The enrollment and initial setup of the PHR allowed for all future transmissions from the remote monitor to the database to flow automatically to the EHR and PHR in near real time. EHR, electronic health record; PHR, personal health record. approximately 90\% agreement. Qualitative analysis software NVivo 11.0 was used to code the transcripts.

\section{Results}

Participants' ages ranged from 36 to 86 years, with a mean (SD) of 67 (14) years, and were predominately male (76\%). All participants were white with the exception of one participant of Hispanic or Latino ethnicity. All study participants received one to three total ICD data summaries. Participants logged in to their PHR between 2 and 17 times (median 5; IQR: 3 ) over the study period ( - Table 1 ). During the study period, one participant received ventricular therapy (shock or antitachycardia pacing).

Six main themes emerged from the interview analysis; see-Appendix $B$ for the list of themes and descriptions of the content in each theme. We present select quotes from 13 unique individuals to characterize these themes and provide examples.

\section{Theme 1: ICD-Related Questions and Concerns}

When participants spoke about their device, over one-third of the group $(n=8)$ spoke about it in reference to receiving (or not receiving) shocks and/or what that might feel like. They had questions regarding physical sensations with their device or heart rhythm. Some participants were concerned 
Table 1 Participant characteristics $(N=21)$

\begin{tabular}{|c|c|c|c|c|c|c|}
\hline Participant & Gender & Age & Education level & $\begin{array}{l}\text { Years } \\
\text { RM }\end{array}$ & $\begin{array}{l}\text { ICD data } \\
\text { summaries }\end{array}$ & PHR logins \\
\hline 1 & M & $36-45$ & High-school graduate/GED & 5 & 1 & 3 \\
\hline 2 & M & $56-65$ & Trade/some college & $<1$ & 2 & 2 \\
\hline 3 & $\mathrm{~F}$ & $46-55$ & High-school graduate/GED & 1 & 2 & 5 \\
\hline 4 & M & $76-85$ & Trade/some college & 3 & 1 & 7 \\
\hline 5 & M & $86-95$ & Postgraduate degree & 2 & 1 & 4 \\
\hline 6 & M & $36-45$ & Post-graduate degree & $<1$ & 2 & 2 \\
\hline 7 & $\mathrm{~F}$ & $66-75$ & High-school graduate/GED & $<1$ & 3 & 7 \\
\hline 8 & $\mathrm{M}$ & $56-65$ & College graduate & 1 & 1 & 3 \\
\hline 9 & M & $66-75$ & Trade/some college & 4 & 1 & 5 \\
\hline 10 & M & $76-85$ & Postgraduate degree & $<1$ & 1 & 7 \\
\hline 11 & $\mathrm{~F}$ & $36-45$ & Postgraduate degree & $<1$ & 2 & 6 \\
\hline 12 & $\mathrm{~F}$ & $66-75$ & No information & 2 & 1 & 6 \\
\hline 13 & $\mathrm{M}$ & $56-65$ & High-school graduate/GED & 5 & 1 & 17 \\
\hline 14 & M & $66-75$ & Postgraduate degree & $<1$ & 2 & 4 \\
\hline 15 & M & $66-75$ & Trade/some college & 5 & 2 & 7 \\
\hline 16 & M & $66-75$ & High-school graduate/GED & 2 & 1 & 4 \\
\hline 17 & M & $66-75$ & Trade/some college & $<1$ & 1 & 4 \\
\hline 18 & $\mathrm{M}$ & $66-75$ & High-school graduate/GED & 3 & 1 & 6 \\
\hline 19 & $\mathrm{M}$ & $76-85$ & High-school graduate/GED & 4 & 2 & 6 \\
\hline 20 & $M$ & $66-75$ & Trade/some college & $<1$ & 1 & 4 \\
\hline 21 & $\mathrm{~F}$ & $56-65$ & Trade/some college & $<1$ & 1 & 7 \\
\hline
\end{tabular}

Abbreviations: F, female; GED, general educational development; ICD, implantable cardioverter defibrillator; M, male; PHR, personal health record; $\mathrm{RM}$, remote monitoring.

because they did not know what type of activity might cause the ICD to fire.

I didn't know how easy or difficult it would be to get myself into a situation where the ICD would have to function. That was the big problem [post-implant] [p02: male, 5665 years old]

Another participant wanted information about anti-tachycardia pacing (ATP) because he knew what shocks felt like, but not ATP.

[ATP would be] probably more important than number of shocks because, if I'd had any shocks I'd know. Uh I've had two and I knew it both times" [p14: male, 66-75 years old]

\section{Theme 2: Perceptions and Understanding about Remote Monitoring}

Overall, the study participants described their experiences with remote monitoring as favorable and positive. For example, nearly half $(n=9)$ of the participants appreciated the convenience of remote monitoring: how little they have to do, the efficiency of it, and how it reduces the number of in-office checks. Additionally, nearly half $(n=10)$ of the participants stated that they perceived remote monitoring as reassuring (i.e., that the clinic is observing their heart rhythm and device status).

There's always that possibility that you have some sort of a problem that isn't serious enough for a shock... that's one of the nice things about knowing you have the remote monitor. That there's someone else also paying attention [p02: male, 56-65 years old]

Specifically, reassurance that everything with the heart and device was normal was an important aspect of remote monitoring:

It feels good [to get the follow up letter] because they say "it's ok," everything's normal so I don't have to worry about it. [p01: male, 36-45 years old]

Although participants felt reassured by remote monitoring, some $(n=5)$ demonstrated a general lack of understanding about how remote monitoring works and how often the transmissions occur: 
I guess I don't really understand totally how it works... if you call it and ask for a report or does it do it automatically or... Does it call in on its own at a certain predetermined time or something?... How often do they do it? [p17: male, 66-75 years old]

One participant pointed out that there was no feedback from the monitor to confirm that the monitor was able to connect with the implanted device and send a transmission successfully.

Without a flash or a buzz or an "ok" or something when it completes its download... if it would ding or do something... it may have a different color light, but it doesn't buzz or shake or something to draw your attention to it [p14: male, 66-75 years old]

While some participants had questions about how remote monitoring works, they still felt reassurance in the fact that it allowed the clinic to keep track of their heart and device function.

\section{Theme 3: Reasons for Using the PHR}

Participants shared motivations for why they logged in to the PHR, such as curiosity or general interest $(n=4)$ :

[What inspired you to log in?] Oh just basic nosiness I mean... interest in seeing what it was going to tell me and what I could learn from it [p14: male, 66-75 years old]

Another motivation to log in to the PHR was to find out if anything was going wrong with the device and to be able to make adjustments based on health information recorded in the PHR. Although no one described taking action based on information that they received in the PHR, two people expressed that there might be potential to use PHR data to make adjustments.

I'm very interested if something's going wrong... and I would like to know about it. I love the idea of getting that [health data]... and then you can make an adjustment, whether it's you exercise more, you exercise less, you lose weight, you know... [p08: male, 56-65 years old]

A few participants $(n=3)$ discussed general interest in seeking health information, based on the underlying belief that knowledge is important when it comes to health care and the ability to take action and control over health issues.

Any time you get more information it's good... For you to know how you can control your own health issues and know what to do when things go wrong, I guess. Knowledge is very important... Never too old to learn [p07: female, 6675 years old]

Overall, participants expressed different reasons for logging into the PHR that provided value to them, whether it was to satisfy interest, knowledge, or the potential to act upon the information.

\section{Theme 4: Facilitators and Barriers to PHR Use}

Ability to access the PHR was an essential component of viewing and using the ICD data summary. Some people had difficulty accessing and using the PHR $(n=5)$, while others expressly found it simple and intuitive $(n=11)$. Specific barriers included confusion because either the participants had more than one PHR or they were not aware because they did not receive notification that an ICD data summary was ready to be viewed in the PHR.

I thought I would be... more active... I just set this aside and didn't do anything, you know, I sort of forgot about it... to tell you the truth. So I thought maybe I'd be getting some calls or... getting some emails or something. [p10: male, 76-85 years old]

Facilitators included ease of use of the PHR for participants who found navigation of the system intuitive. For participants who did not use computers or the Internet, having social support (e.g., friend, family member, or caregiver) facilitated PHR use.

\section{Theme 5: Insufficiencies of the ICD Data Summary}

Several patients $(n=8)$ provided feedback that the sheer volume of information provided in the "View Additional Data" part of the ICD data summary was overwhelming. The widget display with a snapshot of information was more effective than the longer report.

I think this would be great if I could get this information [in the widget]... But that big long page I wouldn't be interested... A few things but not... I wouldn't click on all of those. [p04: male, 76-85 years old]

While some participants did not have as much confidence explaining what they were seeing in the ICD data summary, about half $(n=10)$ shared explicit interest and/or appreciation for having more data as compared with the standard letter.

It was all Greek to me 'cause you got all these abbreviations and stuff on it but... But it was interesting. [p16: male, 6675 years old]

There were also instances $(n=6)$ where participants felt that they should know more and that they could possibly learn how to interpret the information over time. Furthermore, some participants desired a prompt or indication of what to look for in the ICD data summary.

I think it's a neat idea but like I said... I wasn't understanding as much of it as I should. [What do you think could help your understanding?]... I mean if I'd probably just used it more and know what I should be looking for I guess. [p17: male, 66-75 years old]

Still, some participants $(n=6)$ who felt that there was too much information and were not interested in technical details, either because they were assured that the clinic would 
inform them of any issues or they were interested only in limited information.

I'm kind of in the mode of everything happens for me... I can't think of anything I really need to know other than it's working fine. [p18: male, 66-75 years old]

...relevant things I would want to know, not all these technical details. [p10: male, 76-85 years old]

Overall, participants perceived that the information in the ICD data summary was perhaps too extensive and/or complex as displayed in that particular format.

\section{Theme 6: Meaningful Applications for the ICD Data Summary}

The interview provided a platform for participants to share their ideas about how to use or augment the ICD data summary, such as the addition of a glossary or "cheat sheet" in the PHR to supplement the data. Other suggestions included the ability to send messages directly to the clinic and to be able to ask the clinic questions, to see when the next transmission is scheduled to occur, and to receive a text with a link to the PHR when there is an ICD data summary available.

The majority of participants $(n=16)$ spoke about the importance of battery status, seeking a sense of control over when they might need a new battery. Along with battery status, participants' preferences for information included recorded heart rhythms and to be able to track trends.

Maybe like changes in my battery status... if my device is recording anything in the past... 72 hours maybe...? Those are the only two things that (are) at the top of my list that I really wanna know. I wanna like track it, you know what I mean? [p11: female, 36-45 years old]

Two participants suggested that tracking could be useful to associate the date and time of abnormal heart rhythms with activities or environmental factors. In some cases, abnormal heart rhythms are asymptomatic, and the patient does not even notice the occurrence. These participants wanted to use the tracking ability to know when an event occurred of which they were not aware.

If you can tie it [episode] to a date and time I think that would be useful to any person who has a defibrillator. Especially the tachycardia, like I said, 'cause you're going to get zapped, if you get zapped you're not going to forget when that was... You probably could get a date and time and I can tie it to an activity or a stress level. [p06: male, 36-45 years old]

For me personally I wanna see if it started recording, I had an increased heart rate... I want the date, the time, and what the heart rate was, how long it was sustained at that rate, when it came back down... I want the nitty gritty details... just because it blows me away that I don't feel anything. I mean when those times happened, I just, I didn't feel nothing so it just kind of freaks me out. [p11: female, 36-45 years old]
One participant discovered that she could use the ICD data summary to verify that her remote monitor was transmitting successfully. The participant had called the clinic because she was not sure her remote monitor was working. The clinic asked her to initiate a transmission by pushing the button on the remote monitor at home. She followed their instructions, and instead of having to wait for a reply from the clinic, she was able to log in to her PHR and see her ICD data summary from the transmission that she initiated. This provided her with timely reassurance that her remote monitoring was working and transmissions were successfully completed.

\section{Discussion}

This study makes several contributions to applied clinical informatics in cardiology, including insights into patient attitudes and perceptions about receiving ICD data through a PHR. Each of the themes provides specific insights to inform design and development of patient facing interventions for sharing ICD data.

\section{ICD-Related Questions and Concerns}

Participants had questions about how and when their ICD works, particularly if and/or when ventricular therapies occur. Those who had experienced shocks knew what feeling to expect, and those who had not wondered if they would know when a shock was delivered. Research has shown that patients experience fears and anxiety when living with a device and anticipating a shock or trying to prevent a shock from occurring. ${ }^{18}$ Providing educational material and real data as feedback could help patients learn about how the device works for them, mitigating unrealistic fears and making it easier to incorporate into their day-to-day life.

\section{Perceptions and Understanding about Remote Monitoring}

Participants shared questions about when remote monitoring occurs; yet, they felt reassured that someone is monitoring their device and appreciated how easy it is to use remote monitoring. Prior research has shown that empowering individuals with knowledge about their ICD can enhance the patient experience. ${ }^{19}$ Providing ICD data in a PHR could be used as an opportunity to serve as timely feedback that the remote monitor is working, while simultaneously improving patient understanding of how remote monitoring works and what type of data are being captured by the device. However, providing further explanation and discussions surrounding the data (vs. simply pushing the data to the patient) may be necessary to enhance understanding.

\section{Reasons for Using the PHR}

The reasons for logging into the PHR included general curiosity and knowledge-seeking behavior. This may be partly due to the novelty of the PHR and the ICD data summary mechanism. Without prior exposure or experience with using a PHR or accessing ICD data in the PHR, participants may not have been equipped to establish specific goals 
or objectives for using the mechanism, and may have lacked a specific motivation or purpose for logging into the PHR. Still, some envisioned potential for how to use ICD data, such as keeping track of battery status and linking date and time of episodes to activities or stress levels.

\section{Facilitators and Barriers to PHR Use}

The findings surrounding PHR use align with early work exploring potential strategies for PHR adoption. ${ }^{20}$ The sociotechnical-related factors elicited in our findings, such as access to technology and various PHRs, caregiver support, and ability to navigate the PHR interface (some perceived it as easy compared with others), are factors which may influence PHR adoption. While technology allows for efficient delivery of discrete ICD data to patients, it is important to consider sociotechnical factors and usability issues that accompany deployment of novel technologies.

\section{Insufficiencies of the ICD Data Summary}

Our findings support previous research that some individuals were interested in receiving data from remote monitoring, and yet some believed it would be too much information. ${ }^{21}$ Research with patients who have diabetes showed that patients across all levels of education preferred information that was accessible and easy to understand. ${ }^{22}$ Similarly, participants in our study desired information that was easy to understand regardless of education level. Simplicity was important in terms of the terminology used and the amount of data provided. Ideas to simplify the content included removing technical details and providing only relevant information. The meaning of "relevant information" to individual patients, other than knowing that their device is working, requires further investigation. These findings were further indication that patients feel confidence and reassurance with the remote monitoring process and clinic follow-up. Participant ideas to enhance understanding of the detailed information included providing a glossary or supplemental explanation to go with the ICD data summary.

\section{Meaningful Applications for the ICD Data Summary} Participants shared ideas for how to use ICD data. For example, many patients expressed the importance of battery status; these data could be featured in a tailored dashboard along with supporting information that facilitates data interpretation. A few participants thought it would be valuable to track their data from remote monitoring along with other health information from wearable trackers or records in a personal diary to provide context for their ICD data. For example, one participant wanted to be able to track heart rate and episodes and compare them to activities he was doing at the date and time of the episode. Two participants aged between 36 and 45 years were particularly interested in tracking heart rate and date and time of episodes. As the participants in this study ranged in age between 36 and 86 years, it is worth noting that the interest in more complex data tracking seemed to be more common among younger participants. This study reveals the need for tailoring information, which is supported by previous research suggesting that without attention to individual needs, interventions designed to improve engagement with ICD data may not be effective. $^{23}$

\section{Real-World Implications}

The ICD data summary was delivered to participants in a realworld, busy cardiology clinic, providing insight as to how this tool could be incorporated on a larger scale. The transmission of data from remote monitoring to the PHR required no additional workload for the clinic. However, there remain concerns about delivering large amounts of data to patients, as they may not understand and experience anxiety. We did not observe an increase in phone calls to the clinic during this study. ${ }^{11}$ We are examining this concern further in another larger trial. The type of data displayed in the ICD data summary was the consensus opinion of the Heart Rhythm Society and Principal Investigator of the study, who is an implanting and monitoring physician. As described in the findings, patients were interested in receiving the data for their own curiosity, knowledge, and awareness. Important data elements such as battery status, lead status, and number of therapies have the potential to inform patients in a timely manner to provide reassurance and support decision making (e.g., scheduling follow-up visits with the clinic for device adjustment). Future work will focus on redesigning the patient interface and presentation of the data to patients. With an improved design and mechanism for providing meaningful ICD data to patients, we can better understand the impact of delivering ICD data to patients on adherence to remote monitoring, adherence to medication, patient understanding of the continued need for a device, and frequency of clinic and emergency department visits.

\section{Summary}

Simply providing patients with their ICD data in a PHR is not necessarily helpful or effective for enhancing patient understanding and experience. This is consistent with previous work that providing patients with a PHR does not necessarily increase patient engagement. ${ }^{24}$ By employing the ICD data summary as a technology probe, we were able to capture patient perceptions and attitudes about receiving ICD data and gather insights for how to make the mechanism meaningful. We found that some patients wanted guidance to know which data were important and how to use the information. Others had specific ideas for how they wanted to use ICD data, such as to check battery status and/or track changes in data over time. We found that patients have general questions about their device functioning and remote monitoring. Sharing ICD data (including the amount, type, and format) requires tailoring for each individual and may require more supporting information that is not directly related to the data captured by the device. Many studies have pointed to the importance of tailoring the design of PHRs with a focus on supporting a specific health action to promote patient engagement. ${ }^{20,25-27}$ Technology tools such as the PHR interface are appropriate for the purpose of sharing data, as they make personalization and customization of data displays feasible. Barriers to using the technology that we found in this study could potentially be resolved 
through learning and use over time; improved usability; and support from caregivers, family, and friends.

\section{Limitations}

While this was not a design study, we acknowledge that our findings are limited by not involving patients in the design and development of the interface. Rather, we intentionally used the patient interface (ICD data summary) as a technology probe to elicit important insights from participants. These insights can inform future design work and presentation of ICD data to patients. Another limitation is that the ICD data summary did not fully integrate all data elements, such as details about episodes; therefore, we were unable to obtain feedback from participants about every possible data element. One of the functionalities of the PHR used in this study was an email notification triggered by any update in the PHR; however, this mechanism did not work and was not resolved during the study. Another limitation was that the data were integrated from one device vendor; and while the technical feasibility of delivering device data is possible from all vendors, the data from separate vendors may display differently on the interface depending on the specific proprietary data formatting.

\section{Conclusion}

The study provides valuable insights from patient experiences with receiving ICD data from remote monitoring through a PHR. We found that patients have a desire for information that provides reassurance, is easy to understand, and is presented in a meaningful way. These findings can inform future design and development of interfaces for presenting device data to patients. In a larger study that builds on this work, we addressed some of the patients' suggestions in the design of the interface.

\section{Clinical Relevance Statement}

Advancements in technology allow for the delivery of ICD data from remote monitoring to a PHR. There is potential to cultivate engagement by providing meaningful remote monitoring data to patients. The findings from this study suggest that the amount and type of information must be tailored to the individual, thereby providing reassurance and understanding of their device and condition. Tailoring can be accomplished through the design of a patient facing dashboard that allows the individual to select the data elements that are most valuable to them. While we have not tested this solution, it is technically feasible, as modern technologies can provide this type of customizability.

\section{Multiple Choice Question}

The study findings suggest that which of the following aspects of remote monitoring are most important to individuals with ICDs:
A. Peace of mind and reassurance
B. Feedback and follow-up
C. Knowledge and understanding
D. All of the above

Correct Answer: The correct answer is D, all of the above. Some of the participants in the study emphasized the importance of knowledge and understanding, while others stressed the importance of feedback from the remote monitor and/or the clinic. Participants favored the peace of mind and reassurance that remote monitoring provided over inclinic follow-ups only. This suggests that individuals with ICDs undergoing remote monitoring may find any or all of these aspects to be key features of remote monitoring.

\section{Protection of Human and Animal Subjects}

The study protocol and materials were submitted and approved by Parkview Health's Institutional Review Board, part of Parkview Health Systems in Indiana, United States.

\section{Conflict of Interest}

Michael J. Mirro has research funding from the agency for health care research and quality (AHRQ) and the following financial relationships with industry to disclose: research grants from Biotronik, Inc., Medtronic, Inc., and Janssen Scientific Affairs, LLC; consulting fees/honoraria with McKesson Corporation, iRhythm Technologies, Inc., and Zoll Medical Corporation; and financial partnership with Medical Informatics Engineering, outside the submitted work. Dr. Michael J. Mirro's relationships with academia include serving as trustee of Indiana University and on the Indiana University Health Board. Dr. Tammy Toscos has research funding from the agency for health care research and quality (AHRQ) and the following financial relationships with industry to disclose: research grants from Biotronik, Inc., Medtronic, Inc., and Janssen Scientific Affairs, LLC; and iRhythm Technologies Inc. outside the submitted work. Dr. Romisa Rohani Ghahari is also a co-principal investigator on a research grant from Medtronic, Inc.

\section{Acknowledgments}

The authors gratefully acknowledge the patients who participated in this study and shared their experiences. Jeff Donnell, president of NoMoreClipboard, Inc.; Doug Horner, founder and CEO, CTO of Medical Informatics Engineering; and the team at St. Jude Medical for their work on the development of this project. The PHR and ICD summary images represent the products of NoMoreClipboard, Inc. The authors also acknowledge Stan Crosley, JD, director of Indiana University Center for Law, Ethics and Applied Research (CLEAR) in Health Information for guidance on the privacy and security of device data transmission, and Dr. Michael Jackson, Earlham College, for guidance in qualitative research methods. Funding and support provided by the Office of the National Coordinator for Health Information Technology (grant number EP-HIT-10-002), NoMoreClipboard, Inc., Medical Informatics Engineering, and St. Jude Medical.

\section{References}

1 Crossley GH, Boyle A, Vitense H, Chang Y, Mead RH; CONNECT Investigators. The CONNECT (Clinical Evaluation of Remote Notification to Reduce Time to Clinical Decision) trial: the value of 
wireless remote monitoring with automatic clinician alerts. J Am Coll Cardiol 2011;57(10):1181-1189

2 Marcus AD, Weaver C. Heart gadgets test privacy-law limits. Wall St J 2012

3 Hugo Campos. Fighting for the right to open his heart data: Hugo Campos at TEDxCambridge 2011. Available at: https://www. youtube.com/watch?v=oro19-15M8k\&t=18

4 Skov MB, Johansen PG, Skov CS, Lauberg A. No news is good news: remote monitoring of implantable cardioverter-defibrillator patients. CHI'15 2015:827-836

5 Andersen TO, Andersen PRD, Kornum AC, Larsen TM. Understanding patient experience: a deployment study in cardiac remote monitoring. Pervasive Health Conference 2017

6 Petersen HH, Larsen MC, Nielsen OW, Kensing F, Svendsen JH. Patient satisfaction and suggestions for improvement of remote ICD monitoring. J Interv Card Electrophysiol 2012;34(03):317-324

7 Ottenberg AL, Swetz KM, Mueller LA, Gerhardson S, Mueller PS. "We as human beings get farther and farther apart": the experiences of patients with remote monitoring systems. Heart Lung 2013;42(05):313-319

8 Matlock DD. Big Brother is watching you: what do patients think about ICD home monitoring? Circulation 2010;122(04):319-321

9 Woods SS, Schwartz E, Tuepker A, et al. Patient experiences with full electronic access to health records and clinical notes through the My HealtheVet Personal Health Record Pilot: qualitative study. J Med Internet Res 2013;15(03):e65

10 Jabour A, Jones JF. Facilitators and barriers to patients' engagements with personal health records: systematic review. International Conference on Universal Access in Human-Computer Interaction; 2013;472-481

11 Daley C, Allmandinger T, Heral L, Toscos T, Plant R, Mirro M. Engagement of ICD patients: direct electronic messaging of remote monitoring data via a personal health record. EP Lab Digest 2015;15(5). Available at https://www.eplabdigest.com/ articles/Engagement-ICD-Patients-Direct-Electronic-MessagingRemote-Monitoring-Data-Personal-Health

12 HealthIT.gov: What is a personal health record? May 2, 2013. Office of the National Coordinator for Health Information Technology. Available at: https://www.healthit.gov/providers-professionals/ faqs/what-personal-health-record. Accessed July 15, 2017

13 ACC/HIMSS/RSNA. IHE Technical Framework Supplement - Implantable Cardiac Device Interrogation; 2006. Available at: http:// www.ihe.net/Technical_Framework/upload/IHE-CARD-TF-SuppIDCO_PC_2006-04-17.pdf

14 Berg B, Lune H, Lune H. Qualitative Research Methods for the Social Sciences, Vol. 5. Boston, MA: Pearson; 2004
15 Jaspers MW, Steen T, van den Bos C, Geenen M. The think aloud method: a guide to user interface design. Int J Med Inform 2004; 73(11-12):781-795

16 Strauss A, Corbin J. Basics of Qualitative Research. Newbury Park, CA: Sage; 1990

17 Bradley EH, Curry LA, Devers KJ. Qualitative data analysis for health services research: developing taxonomy, themes, and theory. Health Serv Res 2007;42(04):1758-1772

18 Kamphuis HC, de Leeuw JR, Derksen R, Hauer RN, Winnubst JA. Implantable cardioverter defibrillator recipients: quality of life in recipients with and without ICD shock delivery: a prospective study. Europace 2003;5(04):381-389

19 Kuhl EA, Sears SF, Conti JB. Internet-based behavioral change and psychosocial care for patients with cardiovascular disease: a review of cardiac disease-specific applications. Heart Lung 2006;35(06):374-382

20 Tang PC, Ash JS, Bates DW, Overhage JM, Sands DZ. Personal health records: definitions, benefits, and strategies for overcoming barriers to adoption. J Am Med Inform Assoc 2006;13(02):121-126

21 Liu E. Barriers to remote monitoring in patients with implantable cardioverter defibrillators. Yale Medicine Thesis Digital Library (paper no. 1898); 2014. Available at: http://elischolar.library.yale. edu/ymtdl/1898

22 Longo DR, Schubert SL, Wright BA, LeMaster J, Williams CD, Clore JN. Health information seeking, receipt, and use in diabetes selfmanagement. Ann Fam Med 2010;8(04):334-340

23 Habibović M, Denollet J, Cuijpers P, et al. E-health to manage distress in patients with an implantable cardioverter-defibrillator: primary results of the WEBCARE trial. Psychosom Med 2014; 76(08):593-602

24 Toscos T, Daley C, Heral L, et al. Impact of electronic personal health record use on engagement and intermediate health outcomes among cardiac patients: a quasi-experimental study. J Am Med Inform Assoc 2016;23(01):119-128

25 Brennan PF, Downs S, Casper G. Project HealthDesign: rethinking the power and potential of personal health records. J Biomed Inform 2010;43(5, Suppl):S3-S5

26 Ross SE, Johnson KB, Siek KA, Gordon JS, Khan DU, Haverhals LM. Two complementary personal medication management applications developed on a common platform: case report. J Med Internet Res 2011;13(03):e45

27 Siek KA, Ross SE, Khan DU, Haverhals LM, Cali SR, Meyers J. Colorado Care Tablet: the design of an interoperable Personal Health Application to help older adults with multimorbidity manage their medications. J Biomed Inform 2010;43(5, Suppl): S22-S26 


\section{Appendix A}

Semistructured interview guide and prompts for facilitating the interview process. The interview guide was used to ensure that key objectives were met while allowing space and freedom for participants to discuss what came up naturally around these topics.

1. Describe your experience with home monitoring in general over the past 3-4 months.

- Have you had any good (positive) experiences? Could you provide examples?

- Have you had any bad (negative) experiences? Could you provide examples?

2. Have you been receiving any additional information about your device over the past 3-4 months? If yes:

- How do you feel about it?

- Have you had to take any action based on the information you have received?

3. On the baseline survey you mentioned that you would like to receive information about your implantable cardioverter defibrillator (ICD) by email, phone, etc.

- Could you speak more about that?

- Has your opinion changed?

4. During the past 3-4 months you may have received an ICD data summary in your personal health record (PHR). You received some information about this at the beginning of the study.

- Have you been able to access your data summary?

- If no: Do you have any idea why you were not able to?

- If yes:

- How has your experience been accessing the data summary in your PHR?

- How did/does the experience compare with your expectations?

- What information have you received from your data summary?

- Were you able to understand what the information meant?

- What information (if any) have you received in a letter?

- How are the letter and ICD data summary in the PHR different?

- How have you used the information from your ICD data summary?

5. Prompts during the "Think Aloud" protocol:

- Describe what you are looking at or reading.

- Is the process simple or complicated? Please explain.

- What would you change about the display?

6. Follow-up on baseline survey questions:

- How do you feel about any concerns that you had on the baseline survey now that you have used the ICD data summary or know more about it?

- How did the ICD data summary compare with the expectations you had on the baseline survey?

\section{Appendix B}

List of predominant themes, with representative topics provided for each theme, from participant interviews about their experience with remote monitoring, accessing the PHR, and reviewing and using the implantable cardioverter defibrillator (ICD) data summary in the PHR.

Theme 1: ICD-related questions and concerns:

- Unknowns about shocks.

- Unknowns about when the ICD "activates."

- Physical sensations.

Theme 2: Perceptions and understanding about remote monitoring:

- Trust and reassurance that someone is looking after their device and heart.

- Convenience (as compared with in-office checks).

- Unknowns about how remote monitoring works.

- Lack of feedback from the remote monitor.

Theme 3: Reasons for using the personal health record(PHR):

- Curiosity.

- To see information and know if something is wrong.

- To see information because knowledge (in general) is important.

Theme 4: Facilitators and barriers to PHR use:

- Facilitators:

- Ease of use and intuitiveness.

- Support system (informal caregiver).

- Comfort using technology.

- Barriers:

- Accessing alternate PHR.

- Difficulty logging in or navigating the portal.

- Lack of notification or prompts to check the PHR.

- Lack of comfort with technology.

Theme 5: Insufficiencies of the ICD data summary:

- Too much information.

- Did not make sense.

- Not necessary (if anything was wrong, the clinic would call and inform).

- Interesting and neat idea.

- Requires more training or assistance.

Theme 6: Meaningful applications for the ICD data summary:

- Track changes in battery status over time.

- Track changes in heart rhythms over time.

- Provide an indication of abnormal rhythms that are asymptomatic.

- Associate date and time of arrhythmias with activities or stress levels.

- Use as confirmation that the remote monitor is working.

- Provide an indicator of overall health status.

- Add a glossary or cheat sheet for educational information.

Erratum: Erratum: The article has been corrected as per Erratum published on February 28, 2019. DOI of the Erratum is $10.1055 / \mathrm{s}-0039-1681075$. 